Open Access

\title{
Democratizing education at the margins: faculty and practitioner perspectives on delivering online tertiary education for refugees
}

Thomas M. Crea ${ }^{1 *}$ and Neil Sparnon ${ }^{2}$

\footnotetext{
* Correspondence: creat@bc.edu ${ }^{1}$ School of Social Work, Boston College, 140 Commonwealth Ave, Chestnut Hill, MA 02467, USA Full list of author information is available at the end of the article
}

\begin{abstract}
Online distance learning is rapidly becoming a mainstay in higher education. Yet, there still exists unequal access to internet technology among the world's most vulnerable populations. This article reviews the implementation of an online pilot program that provided tertiary education to refugees in Africa and the Middle East, using a unique blend of brick-and-mortar and virtual instruction. Faculty experiences mirrored much of the experiences of instructors in more traditional online education - while onsite staff provided a unique perspective on the embedded nature of the program, based in local contexts. The results of this study helped point the way towards important program modifications to increase the quality of faculty communication and the cultural relevance of the curriculum. Future research is needed to identify whether such programs lead to improved outcomes for refugees.
\end{abstract}

Keywords: Distance education, Higher education, Refugees, Sub-Saharan Africa, Digital capital

\section{Introduction}

Online distance learning is rapidly becoming a mainstay in higher education (Oncu \& Cakir, 2011). These emerging approaches hold promise as a means of democratizing education (Blessinger \& Anchan, 2015), by opening pathways to learning for marginal populations who might otherwise be excluded from such opportunities (Bhatti, Tubaisahat, \& El-Qawasmeh, 2005). Yet, there still exists unequal access to internet technology among the world's most vulnerable populations who lack the "digital capital" to access online educational resources (Seale, Georgeson, Mamas, \& Swain, 2015).

The absence of digital capital can be one aspect of social exclusion (de Haan, 1998) in which individuals or groups are marginalized and prevented from full participation in society. Few groups are perhaps more socially excluded than refugees, who are at particularly heightened risk of marginalization from their host countries (Correa-Velez, Gifford, \& Barnett, 2010). Often living in camps, or increasingly in urban areas, refugees face limited access to adequate education at all levels and opportunities to higher education are especially lacking (Wright \& Plasterer, 2010). The purpose of this article is to review the implementation of an online program which, during its pilot phase, 
provided tertiary education to refugees - Jesuit Commons: Higher Education at the Margins (JC:HEM) -and to explore the perspectives of international volunteer faculty and onsite staff on the benefits and drawbacks of the program.

\section{Distance learning}

The emergence of online distance learning has widened access to higher education and addresses the universal demand for higher education by expanding access to experts, curriculum, and learning materials (Bhatti et al., 2005). This method of instruction can enable students and instructors to use a wide range of internet-based tools to communicate, collaborate, and share resources. Similarly, some students and teachers prefer online collaboration with peers, as it eliminates some of the inhibitions present in face-to-face classrooms (Rhema \& Miliszewska, 2010).

Distance learning has also promoted significant shifts in teaching methodologies (Boling, Hough, Krinsky, Saleem, \& Stevens, 2012). Boling et al. (2012) found that students participating in online learning environments most appreciated social interactions and networking, while instructors were challenged to reconceptualize their own communication to become more bidirectional with students and inclusive of other perspectives. Similarly, in a study of student engagement, Holley and Oliver (2010) concluded that students' voices could be lost when the circumstances of their lives interrupt their full participation in online learning.

These findings also reflect the prescient predictions of an early study, which argued that the role of lecturer in online education would shift towards becoming a "learning catalyst" and "knowledge navigator" (Volery \& Lord, 2000, p. 216) such that studentteacher relationships would become more horizontally balanced. The rapid proliferation of online courses has helped promote epistemological shifts towards more democratic and horizontal relationships among teachers and learners (Krutka, Bergman, Flores, Mason, \& Jack, 2014) particularly at the university level (Zeichner, Payne, \& Brayno, 2015). Yet, Blin and Munro (2008) also found that implementation of online learning at one university failed to change teaching practices significantly, with the main content of courses being driven by "static" resources such as web pages or lecture notes (p. 488). With the rapid expansion of internet-based education, these and other challenges have emerged in effectively employing these tools for students' education.

\section{Challenges of distance learning}

Debate exists regarding the design and implementation of online distance education content, particularly as technological resources and information are being exported from Western universities to other cultural contexts and locations around the world (Rogers, Graham, \& Mayes, 2007). Research has revealed such barriers as social and cultural issues with instructional design, the cultural adaptability of learning materials, and culturally appropriate transformation of courses (McLoughlin \& Oliver, 2000; Rogers et al., 2007). Even in the best of circumstances, attention must be given to the complexities of computermediated instruction and technology mismatches (Liaw, 2006). Students connecting across borders and cultures are also vulnerable to disparities in technology access and skills (Holley \& Oliver, 2010). Advanced software and broadband Internet connections commonly used in the U.S. may not be available to students in less technologically developed countries. 
Perhaps the most significant challenge for international online education is the need for instructional designers to be sensitive and responsive to cultural differences (Rogers et al., 2007). Teachers must be able to understand the full complexity of the instructional context to educate their students effectively. For example, the education needs of youth in refugee camps range far beyond the scope of what is included in traditional education systems, such as issues related to psychosocial challenges, health, landmine awareness, education for peace and citizenship, and specific vocational skills, making it difficult for teachers unfamiliar with daily life in refugee camps to deliver appropriate education (Cooper, 2005). Given these unique dynamics, cross-cultural issues must be addressed to provide quality and relevant instruction, as distance education increasingly moves to an international scale (Rhema \& Miliszewska, 2010).

\section{Faculty perspectives on distance learning}

The success of distance education programming depends greatly on the skill and commitment of the teachers and facilitators who are directing those experiences (Bernard, de Rubalcava, \& St. Pierre, 2000), even as their roles evolve towards knowledge facilitation (Volery \& Lord, 2000). Yet, while there is a prominent focus on students' perceptions of teaching techniques and online pedagogies, the attitudes and experiences of online instructors remain relatively unexplored.

Existing research paints a decidedly mixed picture. One study of impediments to blended instruction (i.e., a combination of face-to-face and online instruction) found that teachers experienced significant difficulties in managing the complexities of blended courses. Among these complexities were difficulties in managing communication with students; increased time required to coordinate the course; and challenges in navigating the technology required (Ocak, 2011). These findings were reflected in another study of online learning environments, where text-based content tended to be emphasized over teacher-student interactions, although students most appreciated the social aspects of the course (Boling et al., 2012). Krutka et al. (2014) found similar obstacles to navigating online reflection among pre-service teachers, but also found that these teachers valued peer feedback and the collaborative aspects of online communication.

The challenges of teaching online courses can be mitigated by preparation support, and dedicated preparation time. In a series of studies of faculty's experiences with distance education, faculty found it difficult to manage multiple learning sites (Cook, Ley, Crawford, \& Warner, 2009). Consequently, faculty expressed the need for early and appropriate training for unfamiliar teaching experiences, particularly regarding technological and cross-cultural support in order to develop competencies in guiding international online distance courses (Cook et al., 2009). Faculty workloads at universities also typically do not permit faculty members to spend many hours learning how to design, develop, and teach an online distance course on their own. Without instructional support, another study found that faculty members believed it unreasonable to require them to be prepared to fulfill all of the roles expected of them as online distance educators (Easton, 2003).

Distance education has expanded both the potential scope of student populations, and challenged traditional notions of pedagogy and the nature of teacher-student interactions. Yet, very little research on distance education has focused on providing higher education to highly vulnerable and marginalized populations. In the next section, we 
describe the pilot phase of Jesuit Commons: Higher Education at the Margins (JC:HEM) which, from 2010 to 2014, provided tertiary education to refugees via an online delivery system.

\section{Jesuit Commons: Higher Education at the Margins}

The JC:HEM pilot project commenced in September 2010, in partnership with Jesuit Refugee Service (JRS), following a needs assessment which documented a demand for tertiary education among the refugee population. JC:HEM was invited by JRS to serve refugees living in Dzaleka camp in Malawi, Kakuma camp in Kenya, and urban refugees living in Amman, Jordan. An original pilot site included Aleppo, Syria, but implementation at this site was postponed during the war. The pilot phase was guided by the principles of sustainability, scalability, and transferability, using technology to identify program structures in keeping with these principles (Jesuit Commons: Higher Education at the Margins [JC:HEM], 2010). After identifying needs for tertiary education amongst refugee populations, the JC:HEM program leveraged the increased availability of technology and improved internet connectivity across Africa to implement distance learning in the pilot sites (Dankova \& Giner, 2011). The pilot phase of JC:HEM concluded in December 2013 and entered a 2-year capacity-building phase. JC:HEM now operates under the name Jesuit Worldwide Learning (JWL, 2017).

\section{Program design and delivery}

During the pilot, JC:HEM offered two awards: the Community Service Learning Track (CSLT) and the Diploma. CSLTs were delivered in conventional onsite classroom environments. They were developed in response to the stated needs of local communities and covered subjects as diverse as psychosocial case management, business skills, community development, and others. Curricula and teaching strategies for each track were developed by onsite staff with the assistance of JC:HEM International Faculty volunteers for subsequent onsite delivery. Typically, they comprised $150 \mathrm{~h}$ of coursework offered over 24 weeks as well as additional field hours. CSLT Facilitators at each site managed admissions and practicum settings.

The Diploma in Liberal Studies comprised 16, 8-week online university-level courses, starting with a zero credit-rated Bridge to Learning course. This was followed by 10 foundational Liberal Arts courses and concluded with a five course 'concentration' in either Business or Education. The Diploma in Liberal Studies was usually studied over 3 years for 45 credits awarded by Regis University.

Students were recruited through a four-phase process developed in partnership with the onsite partner JRS and the awarding institution, Regis University. This process comprised an eligibility check (normally, that the applicant was registered with UNHCR as a refugee); an English test; a proctored essay scored against a rubric by teams of volunteer faculty; and a face-to-face interview. Applicants were required to pass each stage before they could progress to the next, and ultimately were ranked according to weighted essay and interview scores. The final selection admitted as many applicants as space would allow. In addition, in making this final selection, JC:HEM sought to ensure that $10 \%$ of all successful candidates were drawn from the local community and that at least $30 \%$ of all students admitted were female. 
At the outset of the pilot, a JC:HEM Academic Committee was formed to articulate the nature and content of a Diploma in Liberal Studies. This committee produced an outline program comprising 15 8-week courses that would be taken over 3 years. Those institutions which had expressed an interest in supporting the pilot were invited to contribute courses from their existing portfolios. The syllabus of each donated course was assessed by the JC:HEM Academic Committee to ensure that it was at the appropriate level and compatible with the wider objectives of both the Diploma program and the initiative as a whole. In all, seven institutions donated a total of 24 courses. However, not only did this process take time, it was only partially successful. Only a handful of the courses offered proved suitable and, during the first 3 years of the pilot, JC:HEM offered those courses, while seeking additional course donations to complete the program. Towards the end of the pilot, JC:HEM developed courses to fill the gaps that remained.

At the conclusion of the pilot, a review was undertaken that made it clear that JC:HEM could not be reliant on donated courses for several reasons. For example, not all of the courses fitted naturally into academic framework of the Diploma program as developed by the JC:HEM Academic Committee. Others did not work in the context in which they were being offered. Memorably, one course invited students to observe the interactions of customers at their local Starbucks, but there were many other examples where the assumptions inherent in the course design were not transferable. Clearly, to ensure the academic coherence, integrity and credibility of the program, it was necessary not only to redevelop the Diploma program overall, but to draw on JC:HEM's experience and expertise in developing courses to students at the margins to create excellent courses that were sufficiently flexible to work effectively in the numerous and various circumstances in which they would be offered.

Diploma students were typically placed in 'sections' of 15 , each comprising students from all sites, taught online by volunteer International Faculty members using donated courses, and later, courses developed specifically for JC:HEM. Each annual cohort therefore typically comprised between 3 and 8 sections. These communities comprised on the one hand, an online community in which students encountered their peers from other locations, nationalities, backgrounds, faiths and language groups; on the other, a community at their own location to provide peer support, encouragement and assistance. At the end of each 8 week online course, feedback was solicited from students via the appropriate learning management system, and from faculty through the JC:HEM Personal Space Discussion Boards. This feedback was reviewed by the Academic Coordinator and subsequently passed to the Lead Faculty to consider any necessary changes to the course before its next delivery.

Faculty were predominately volunteers drawn primarily from Jesuit universities in the United States who responded to an open invitation disseminated through the mailbase of the Association of Jesuit Colleges and Universities (AJCU). As the pilot progressed, other mechanisms were used to call for volunteers including LinkedIn and similar social and professional networks. These efforts were supplemented by personal visits by JC:HEM staff to higher education institutions in North and South America, Africa, Asia and Europe, as well as presentations at professional and academic conferences and seminars. From 2012, Faculty were also invited to participate in the JC:HEM Faculty Orientation Course which provided details of the program, the learning sites, the 
learning management system, the courses and the students they were about to encounter. By 2013, JC:HEM had established a group of institutional liaisons, comprising more than 20 members, with whom it worked regularly to identify and meet its faculty recruitment needs.

Faculty were organized into Academic Teams under the supervision of Lead Faculty members who coordinated course expectations and strategies. While each Faculty had his or her own section of between 10 and 15 students, the Team was able to coordinate its efforts on matters such as deadlines and extensions, guidance given to students as well as the interpretation of academic regulations on issues such as late papers and plagiarism. As the Lead Faculty was most often someone who had previous experience of teaching the course for JC:HEM, Academic Teams were particularly effective in supporting new Faculty as both the scope and scale of the JC:HEM initiative expanded.

Faculty were trained through a bespoke online course which sought to familiarize them with several aspects of the JC:HEM program. This training included: (a) an overview of the program, its aims, locations, partners, and key staff; (b) a summary of the learning sites, student numbers, the physical facilities available to students and on-site staff; (c) guidance on the use of the learning platform; (d) guidance on the course to be taught, its content, assessment processes; (e) details of the students, their background, language skills and demographics; and (f) rules and regulations and other key documents.

\section{Learning platform, materials, and instructional design}

Initially, JC:HEM's learning platform was dependent on that used by the donating institution on which any given course was held. These platforms included Blackboard, D2L and Moodle. As such, in its pilot phase, JC:HEM required students to hold up to three different login details and to log into each system separately. Over time, all courses were migrated to an instance of Blackboard donated by Georgetown University. Following the 2010-2013 pilot program, all courses were explicitly designed for delivery through the Georgetown Blackboard learning platform.

In the pilot phase of JC:HEM, courses were largely donated by contributing universities such that there was a need to purchase and ship the designated textbooks to the sites. Shipping was not only expensive but posed significant logistical challenges in terms of transportation, legal and customs requirements that frequently resulted in significant delays. JC:HEM experimented with various methods to address these challenges, including the purchase of Kindles onto which texts could be downloaded. Yet, early versions of the Kindle were difficult to use in bright light and often proved too fragile for the hot and rough conditions of refugee camps. Not all of the required texts were available in electronic versions, and when available, texts were not noticeably cheaper per unit. Interestingly, the students themselves expressed a preference for hard copies of the texts - considering them easier to use and more durable. As JC:HEM developed over time, there was a conscious effort to move away from text-based inputs and a greater use of bespoke video and audit inputs. Text, where it was used, often relied upon open-source materials and was incorporated into the online course itself.

The instructional design forming the heart of JC:HEM had been formulated and honed over time. Based on the principles of Jesuit pedagogy developed by St. Ignatius 
of Loyola (the founder of the Jesuit order of Catholic priests), the program sought to incorporate three key elements into each week's work, each course, and the program as a whole: knowledge, reflection and action. This framework allowed instructional designers to contextualize their approach and, in turn, allowed the students to examine their world through an objective lens and contextualize the content for themselves.

For example, initial versions of the program included a course on African history. This course proved unpopular with students who indicated they wanted to learn about the history of other parts of the world, and to develop the skills and tools necessary to discern bias and favor not only in the history course itself but in the world around them. In another example, a popular business course focused on the principles of developing effective business plans and financial planning (knowledge); an invitation to students to consider how these principles might be implemented in their own lives (reflection; i.e., what would and would not work given the circumstances in which they found themselves); and finally, the development and implementation of their own plans (action). Following enrollment in this course, several students began their own business including the creation of a bicycle taxi firm, a restaurant, and painting business.

Course assessment was managed through the learning delivery system and took various forms. Faculty might for example assess a student's input into an online discussion via the discussion board. Other forms of assessment included the creation and production of plays which were subsequently filmed and uploaded for assessment by the online faculty. However, the more conventional forms of assessment - written essays, tests and quizzes, usually electronically produced and uploaded to the learning management system or pre-loaded and taken online - remained the most common approach.

\section{Onsite program infrastructure and information technology}

Although the JC:HEM project was originally envisaged as an online program that would rely on the internet and electronic communications, as it evolved it required increasing levels of building infrastructure at its delivery sites (Sparnon, 2011). Therefore, in addition to internet masts and servers, the model of delivery at all three locations became based around the use of computer laboratories supplemented by office and classroom space. These shifts required significant renovations to allow for onsite teaching space.

The initial JC:HEM assessment team considered that the most effective solution would be to install wireless networks in the camps to enable students to access content irrespective of location. Yet, several factors mitigated against this: first, concerns from the onsite partner around security - it was made clear that the provision of personal computing equipment would make students unacceptably vulnerable in their own homes; second, the unsuitability of many student homes as learning spaces (e.g., no electricity); third, the desire of JC:HEM staff and administrators and the students themselves to form a mutually supportive 'community of learners'; fourth, it became clear that the transition from classroom-based, didactic teaching to a more self-reliant learning style was a significant step for many of the students - and indeed many of the onsite staff. As such it was necessary to provide a bridge to enable students and staff to transition effectively.

The learning centers that therefore emerged (known as the 'Arrupe Center') at each site comprised a computer laboratory which could be connected to the learning 
network - initially via ethernet cables or microwave, but later, wirelessly. The content itself was held on local multipoint servers. In addition, the centers included a large learning space that could be used as a conventional classroom (particularly for CSLT delivery), seminar or group activity learning space, a small library, plus office space for the onsite staff.

Several environmental factors needed attention. Security remained a key issue. All sites were fenced and gated and patrolled by security guards at all times. Opening hours were restricted to those allowed by the JC:HEM site coordinator which, given the size of Kakuma for example (at the time, approximately 124,000 people), often meant that students had to walk several hours to and from the site for a relatively short period of access. This problem was compounded by technical issues around power and internet access and instances of flash flooding which made travel to the site impossible. Other issues have included food riots, cholera outbreaks, security restrictions and curfews implemented in the wake of terrorist attacks in the region.

The program was additionally challenged by issues surrounding the provision of electrical power. A particular concern at Kakuma was the need for air conditioning to enable computers and students alike to function for sustained periods. At refugee camps, therefore, implementation required additional power supplies, often from commercial providers supplemented by solar panels and generators.

\section{Evaluation assessment}

Near the end of the JC:HEM pilot, an expert in outcome assessment was hired by JC:HEM to provide a comprehensive assessment of 2010 to 2013 objectives, and data from that assessment inform this article. The assessment was conceptualized as developmental research, to help "improve an existing instructional model/process" and to assist in providing "pathways of guidelines...for successful implementation" (Oncu \& Cakir, 2011, p. 1103) of JC:HEM in the next phase of implementation, following an iterative process. This final assessment, completed in 2014, also served as a follow up to an interim assessment conducted midway through the pilot, which identified strengths and weaknesses in instructional technology and curriculum content.

\section{Methods}

\section{Sample}

This study followed a two-part sampling methodology, and focused on the perspectives of program staff and international faculty (other studies focused on the perspectives of students themselves and lessons learned from implementation; see Crea, 2016; Crea \& McFarland, 2015). First, JC:HEM/JRS staff were individually interviewed between August and December 2013, either face-to-face, via Skype, or by completing a written survey $(n=23$; see Table 1$)$. Second, all 130 International Faculty were sent an online survey between February and March 2014. Fifty six completed some part of the survey for a response rate of $43 \%$. Of these, between 38 and 44 participants responded to individual survey items.

\section{Measures}

A nine-question interview form was developed to assess staff perceptions and understanding of JC:HEM, particularly its strengths, weaknesses, and impact in the communities in which it has been implemented. During live interviews and focus groups, 
Table 1 List of Onsite Staff Interviewed $(n=23)$

\begin{tabular}{ll}
\hline Site & Role \\
\hline Kakuma & JC:HEM Coordinator \\
& JRS Regional Director \\
& Assistant Education Field Officer \\
& Community \& Business Development Track Facilitator \\
& English Language Learning Facilitator \\
& CSLT Facilitator and Academic Track Coordinator \\
& CSLT Psychosocial Case Management facilitator \\
& Information Technology Assistant \\
Dzaleka & JC:HEM Coordinator \\
& JRS Regional Director \\
JC:HEM Staff (Focus Group, $n=10)$ \\
\\
Jmman & JC:HEM Coordinator \\
& JRS Country Director \\
& CSLT Facilitator \\
\hline
\end{tabular}

responses were typed to be as close as possible to respondents' words. For those staff members who were not able to participate in live interviews or focus groups (most of the Kakuma staff), participants typed their own responses to questions in Word versions of the form and emailed to the principal investigator.

An online survey was developed to assess international faculty's perceptions of and experiences with JC:HEM. The survey contained seven questions pertaining to faculty recruitment, orientation, the academic team, the course taught, and experiences teaching the course. A survey link was disseminated to faculty who have volunteered with JC:HEM. The email included a deadline to return the survey and two subsequent reminder emails were sent. No incentive was offered to potential respondents.

\section{Analysis}

Open-ended survey comments were pooled into the following categories using a deductive approach, prior to coding for specific themes: (1) Program strengths; and (2) Areas for future growth. Qualitative data from staff and were analyzed through an inductive process of assigning codes to transcribed data (Strauss \& Corbin, 1998), to produce a grounded theory of staff perceptions of JC:HEM. Through independent and joint review, three researchers developed and grouped codes into themes for analysis, using open and axial coding. The principal investigator then reviewed these codes for accuracy.

\section{Results}

International faculty perspectives

Program strengths

Researchers identified 41 comments related to strengths of JC:HEM from the perspectives of international faculty. Positive Communication was the most frequently 
identified theme. Some faculty members appreciated the collaboration among the faculty, particularly because of the "ability to ask each other questions," and the ability "to contact my team at any time." Some faculty also identified the information technology platform as a strength, especially Blackboard and the "JC:HEM sites," "continual emailing," and the fact that "the connection via internet... could be accessed through the module." Others appreciated phone contact, the ability to provide feedback about their experience, and general appreciation for communication.

Another theme that emerged from the comments related to faculty members' Commitment to JC:HEM and the students. Some related to a sense of fulfillment in teaching within JC:HEM: "It was an amazing experience and I hope to continue to teach in this program as my time and schedule allows"; "The students and the organization are wonderful and I would happily teach again"; "I learn something from my students in every class. The experience of teaching and making the material pertinent for them continually makes me improve as an educator." Respondents also highlighted team support as a facet of commitment to JC:HEM. Faculty members said, "Fellow instructors and course leaders took the course very seriously and were respectful." Another said, "The commitment of everyone involved created an atmosphere of cooperation and support that helped to address issues and tasks."

Participants also expressed that JC:HEM Leadership was a strength of the program. Particularly, instructors were grateful for the contributions of on-site staff: "Working directly with on-site staff that worked regularly with the students helped me connect better with students' needs." Faculty also appreciated the use of team leaders in instruction: "Communication works when there is a good team leader."

\section{Areas for future growth}

Researchers identified 43 comments related to areas of future growth for JC:HEM. The most prevalent theme among these related to Communication issues. Several faculty perceived a lack of cohesion among team members and a feeling of disconnectedness from teams and the larger JC:HEM program. One faculty member said, "Would like to have felt like I was more connected to the WHOLE of JC:HEM rather than a small corner, needed context for the overall program/organization." Another said, "I did not get the sense that we were working on a team. Faculty shared concerns via email from time to time but there was no real collaborating on how to solve problems." A related problem was the lack of availability of other team members, with other instructors not responding to faculty's comments or questions in a timely way, or at all, even to student contributions. Three comments also related to lack of contact with on-site staff: "Much of the time teaching felt like throwing information over a high wall, not knowing where it was landing."

Another area of concern identified by faculty related to the Resources available to instructors. Nearly half of these comments pertained to the relevancy of instructional materials to students' contexts: "I still believe that work needs to be done on creating content that is applicable to the environment and students' needs rather than attempting to customize an existing course"; "The instructional module at times was not transferable to the camps. I had to make several adjustments so that it would be meaningful for the online students...Ideas from the academic team in terms of past modifications would have been good as well." An additional area of concern related to the amount of time faculty felt they needed to dedicate to the course, given that the position was 
voluntary and their time was not compensated: "Making track changes on four-six papers per student per 8 week session is unreasonable when this course is a volunteer overload and I teach a four-four (courseload)"; "Some of the instructors were so overcommitted that they had minimal time or resources to put into the course. I think the students were the ones to suffer because of this...." Other problems emerged with technology, particularly poor connectivity and its impact on communicating with students.

A third area of future growth identified by faculty was to increase the Clarity around expectations for students, faculty and staff. Faculty members were unclear how to provide a proper assessment for students given the cultural differences between instructors and students, as well as unclear criteria for how to handle these differences. One instructor said, "Grading papers is almost impossible. So many students have poor writing skills that the papers take forever to grade. They also want specific help with English and grammar in addition to grading content." Similarly, another noted, "I had concerns about standards and the credibility of the course outcomes/assessment. It is not the same as it would have been for U.S. students." Another area related to expectations for faculty work and performance. One instructor noted a lack of understanding about communication with on-site staff: "I wasn't told that I could coordinate directly with the onsite staff who worked in the classroom with the students until about halfway through the course...." Others mentioned faculty members who did not fulfill their teaching obligations and created problems for other faculty members: "One lead faculty left the team hanging and just didn't show. I had to take over at one time to save the rest of the team who had not taught before. I cut back a vacation and made it happen"; "I had a few as a team leader who first thought their role was more like 'guest lecturer'."

\section{Onsite staff perspectives \\ Perceived benefits of JC:HEM}

In survey interviews, JC:HEM onsite staff and administrators employed by JRS were asked about their perceptions of the strengths of the program. Researchers identified seven themes related to benefits of JC:HEM: community building, impact, skills, outlook and future orientation, and culture. These are described below.

Community building Staff identified a number of factors related to the creation of a strengthened community as a result of the JC:HEM program (51 comments). According to staff, concrete skills taught at JC:HEM have helped students to improve functioning within their community. One staff person in Kakuma stated that students "often talk very positively about the communication and leadership classes and the ways they've influenced their abilities to effectively communicate with their families and their communities. Many of our students are leaders within the community and use the critical thinking skills they improve upon in their studies on a day to day basis in interacting with and organizing the community." Another staff person in Kakuma cited students as bringing transformational change to their communities by acting "as agents of change through helping others in the community to understand situations, acting on opportunities and working with the available resources to solve issues and have developed most some of the communities in the camp". 
Furthermore, completion of the Diploma and CSLT programs has equipped students with the necessary skills to acquire jobs that help them to better serve their communities. According to a stakeholder in Kakuma "students have started applying the skills in different NGOs as teachers, social workers, work supervisors and managers... many students have improved working conditions at their NGOs and communities." Other students have taken jobs as "health workers, community leaders, translators and interpreters, etc." For fields where there are limited employment opportunities, students "volunteer in respite care...many students are continuing as communications volunteers."

Community cohesion is evidenced through increased unity and peace-building within the program and the communities in the camps. One staff member stated that JC:HEM's "non-discriminatory nature is highly appreciated as a unifying and peace building space." Another noted, "It allows students to talk to people from different countries, different cultures, different language backgrounds and different religions". Students from diverse backgrounds are able to collaborate and "exchange ideas, bring ideas of unity". This opportunity for collaboration, communication, and friendship is not evidenced elsewhere in the camps. Cross-cultural learning, in combination with the skills taught at JC:HEM has helped to increase peace-building amongst students and persons in the communities. A staff member in Kakuma stated that "skills acquired PCM have helped community members in handling community cases hence reducing conflict rate in the community." Another observed, "Some people in the camp were enemies, but through the JC: HEM they come together as brothers and sisters."

Increased participation in community activities can be seen through creation of community-based organizations, employment with social services, creation of and participation in extracurricular activities and creation of service days. After obtaining skills through JC:HEM, many students begin to work for social services within the camps. According to a stakeholder in Kakuma, "the program has played a big role in improving social services rendered by refugees from different agencies to the community." Other students have used the skills they acquired through JC:HEM to start "their own community based organizations" and are running them in the camp or "in their home countries." Some are operating sports leagues in the camp for youth, drama clubs, and various other education activities. Another staff stated that "Through JC:HEM's CSLT courses that provide knowledge and skills, several people in Kakuma have come up with small scale business schemes, CBOs, development groups and creative events that have reduced over dependence on agencies." Furthermore, staff in Dzaleka are trying to increase "the links of education back into the community" by strengthening their "service days" program. This program would ensure that "within every course there is a community based activity and that is linked into the course, like service learning." As one staff member concluded, "We're not just providing a university education in the abstract. We're providing a community university education, so we can't just divorce the context from the whole thing. We'd like to see gains in the now not just the future."

Impact Another positive influence stakeholders identified from JC:HEM was the impact it had on refugees and their communities (38 comments). Impact can be identified through increased educational opportunities, advancement, increased employment, empowerment, 
trauma reduction, and quality of life. By and large, staff viewed increased educational opportunities as a result of JC:HEM participation as having the biggest impact on community dynamics. One staff person said, "Higher education is important for the refugee youth and adults. Every year, Kakuma refugee camp receives a big number of new arrivals, who succeeded to finish their secondary level of education. In addition, the two refugee secondary schools produce more than 100 students who finish high school every year. For these people to be of great help to their societies, they need to advance their studies."

Aside from increased educational opportunities, the advancement that participants made overall in their lives led to an increase in empowerment and overall better quality of life, according to staff. One staff person reported that JC:HEM has "changed their lives, their families status, because of working, things have improved." Another cited that JC:HEM has "improved the quality of life in the camp. The improvement ranges from having primary and secondary school students strive to do well so as to access university studies through JC:HEM, to improved family conversations and community participation and discussions around issues learnt in class, to improved delivery of services to beneficiaries within the NGO community by students participating in the program."

Skill building Another common theme identified by stakeholders as a strength of JC:HEM was the skills which were developed by participants ( 35 comments). Leadership, overall skill building, and practical skill application were all reported as contributing to an overall improved sense of community within JC:HEM sites.

One staff person reported that "many students who have joined the JC: HEM, including myself, have seen a major change in our critical thinking, interpersonal communication, and in using the different leadership skills to improve our societies." Another stakeholder cited a change among younger people, saying, "more people especially the young adults are gaining knowledge and skills to better their lives and that of their families and community at large." A third said that through CSLTs, "several people in Kakuma have come up with small scale business schemes, community-based organizations, development groups and creative events that have reduced over-dependence on agencies." This same person observed that "many of the participants have acted as agents of change through helping others in the community to understand situations, acting on opportunities and working with the available resources to solve issues and develop the communities in the camp." In this way, skill-building also serves the larger community by helping participants be leaders in their communities.

Outlook and future orientation Staff identified JC:HEM as having created a positive outlook on life for participating students (22 comments). Staff in Dzaleka and Kakuma reported that students felt a renewed sense of hope in their future due to their ability to further their education and participate in JC:HEM. A staff person in Kakuma stated "Education has also shown to help build the refugees' courage and hope for the future, as well as helped to reduce the trauma refugees have encountered prior to coming to the camp." Increased access to education has also helped students to open their minds and learn about other cultures. Obtaining newly acquired perspectives, skills and knowledge, helps to increase 
students confidence in themselves and in their communities, allowing them to "look at themselves as people who have achieved something."

This sense of achievement has resulted in feelings of appreciation of and respect for the JC:HEM programs and its participants. As a result there has been an increased interest in participation in the program: "With the view of admiration from the non participants to participants, the number of people acquiring about JCHEM programs and applying for them, has greatly increased due to the importance detected by different communities from the participants' improvement in their services."

Culture Stakeholders identified culture, and particularly cultural understanding (14 comments), as a strength of JC:HEM. "With the kind content in the courses offered by JCHEM, peoples' attitudes towards others' culture and behavior have positively changed ..." one stakeholder reported. Another stakeholder stated that, "JCHEM is like a center where people from different races meet, being educated in different ways." Furthermore, the "diversity of the camp has helped in making the program successful, since it serves as a catalyst for bringing people from different cultures together." Another staff person said that JC:HEM has "given a whole different dimension to camp life."

\section{Perceived challenges to JC:HEM implementation}

Staff members also observed a number of challenges to JC:HEM implementation, particularly related to program delivery, gender issues, lack of resources, availability of postprogram opportunities, demand for limited spots, lack of local certification, and language barriers. These are described below.

Program delivery Challenges related to program delivery emerged as the most significant barrier to implementation (18 comments). Communication between online faculty and sitebased staff made effective delivery of material difficult. One staff member stated: "What has made the planning process difficult is participation of international faculty. This stunted the development process by slowing significantly. They're working on a volunteer basis. But they're not replying to inquiries, and promising materials but didn't deliver." Another staff person said that "because communication (with professors) was so disjointed, it was sometimes hard." This person also observed that the "structure was confusing to know which professor to contact. Sometimes one professor would make a decision for one section but others would not." An additional problem was noted that lead faculty are not consistent so that "discrepancies in grades and feedback occurred that don't relate to the quality of work". This issue was particularly discouraging for students.

Another significant challenge was the mismatch between the curriculum content and the contexts in which students live. One staff member said, "The curriculum felt disconnected from the local reality here. It was hard to find meaning and relevance in various texts and assignments...." Another stated, "I think there's a disconnect (in the curriculum) in terms of the lives the students are living."

Gender issues Another area in which staff observed significant challenges is related to the structural disadvantage for women related to cultures of origin (16 comments). Several staff persons mentioned that household duties and childcare responsibilities impede women from devoting the time necessary to pursue education. One stakeholder 
said "it's due to different cultural norms respected by a set of people coming from different countries, this has resulted in most of the women getting more involved in domestic work than education." Another staff person said "I think that many women are still stuck in the homes, taking care of children, washing and cooking... A woman may find it difficult to leave her chores and her child behind to come to the Lab...." Another noted, "if a man goes to school and woman also goes to school, what are the children going to do? They are thinking to look for their life, not studying."

Given the ingrained cultural barriers of privileging boys over girls in accessing education, there are few female students who finished high school and qualified for this program. One staff member said, "In this camp women are facing a number of challenges that hinder them to continue from primary school education to tertiary. This gives us a limited number to select from."

Lack of resources A commonly cited barrier is a general lack of resources (11 comments), specifically limitations with information technology and access to books. Particularly in the Africa sites, challenges were noted around information technology and internet connections. Some staff noted the problems students experienced in accessing textbooks in a timely manner.

Post-program opportunities Several staff mentioned the lack of post-program opportunities as a significant problem (nine comments). As one staff person observed, "In general, the needs of education are met. The needs of what happens beyond are not....Students who saw education as a path to something are unclear where that path has taken them." Another remarked more pointedly, "Some people like it (JC:HEM) and they would like to know what it offers, but onlookers laugh at the participants, that it is wasting their time since JC:HEM is not taking them anywhere." Another staff person said, "it's impossible for all education needs and desires to be met completely." Another said, "I think that 'what next' is something that should really be thought about."

Demand for limited spots Quotas and class size limitations emerged as another barrier according to staff (nine comments). Several noted that the number of refugees interested in joining the program significantly exceeded the spaces available for enrollment. This problem was evident in both Kakuma and Dzaleka camps. The limited class size also poses challenges in the selection of students because of the needs to balance nationalities. One staff member said, "Sometimes it makes the selection of students somehow harder when it comes to balancing them according to nationality. In this case you may end up including some people who may not have all the qualifications." Another expressed concerns for refugee applicants who are not enrolled "...those that were left do nothing in the camp, doing drinking." The same person also identified the need to expand the program: "they need to dig deep and provide more help, because only a few people are allowed to attend the classes."

Lack of local certification The fact that JC:HEM doesn't provide recognized certification (five comments) is an inherent barrier. One stakeholder said "Some don't see much value because it's not recognized as an official degree." Another stakeholder 
said "part of the reason they may not be employed related to correspondence with (government's) definition of qualification."

Language barriers In addition, language barriers (three comments) are hindering some students from accessing JC:HEM. One stakeholder from Kakuma camp identified refugees' English proficiency as a challenge: "A big number of people who apply for these courses, have problems in understanding English which needs a lot of creativity in making all ends meet." Another stakeholder said "Many interested students don't have English schools to apply to diploma program."

\section{Discussion}

The JC:HEM pilot program initially was conceptualized as primarily a distance learning program (Jesuit Commons: Higher Education at the Margins [JC:HEM], 2010), but as the pilot evolved, the demand for increased physical infrastructure became evident (Sparnon, 2011). In this way, JC:HEM has emerged as a unique blend of brick-andmortar and virtual instruction (Ocak, 2011), with international faculty teaching online, moderated by on-the-ground staff. In spite of the novelty of this approach, the dynamics emerging from faculty experiences mirror much of the experiences of instructors in more traditional online education - while onsite staff provide a unique perspective on the embedded nature of the program, based in local contexts.

International faculty perspectives reinforce the need for adequate orientation and preparation support (Cook et al., 2009). Faculty were generally positive about the appropriateness of information provided during orientation but also expressed the need to receive this information more quickly. This dynamic seems to play out in faculty's use of the Discussion Boards, which they appreciated, yet seemed to lack adequate preparation ahead of time. Having such dedicated preparation (Cook et al., 2009) might help offset the technical challenges often experienced by distance learning faculty (Ocak, 2011).

Faculty generally enjoyed working on academic teams, and similar to previous research (Krutka et al., 2014), viewed the online academic team approach as allowing connections to form as the process built collegiality, supportive relationships, and a network of experts for the program. Yet, significant problems emerged when faculty leaders did not communicate with team members or failed to complete their duties as instructors and course leaders. The issue of clear and frequent communication among faculty is also reflected in the observations of staff - particularly when on-site staff and students are not able to receive clarification on assignments and grading. In fact, the issue of communication was identified by faculty as the main area for JC:HEM future growth, with a desire expressed for greater collaboration among teams and more clearly articulated expectations for instruction - and again highlights the endemic problem of clear and adequate communication in distance learning (Blin \& Munro, 2008; Boling et al., 2012).

Faculty were critical of course content as it related to cultural context, cultural sensitivity, and availability of resources. These findings reflect a common challenge to creating content for distance learning, particularly for international populations (Cooper, 2005; Rogers et al., 2007). These observations also are in line with those made by onsite staff, 
who struggled at times to make course content relevant to the context of students' lives. These critiques likely also are a function of the donated nature of the courses, and the limited staff time available to tailor course content to unique contexts so that the curriculum was not tailored specifically to the learners' needs.

In contrast to faculty perspectives, onsite staff tended to focus on the impact of JC:HEM participation on students' lives beyond the classroom. Most onsite staff stated that the skills acquired through JC:HEM have allowed students to make an impact in their community through the creation of extracurricular activities, and working as teachers, social workers, health workers, translators, and community leaders. Nearly half of staff reported that JC:HEM serves as a unifying space that allows students from different countries, cultures, language backgrounds and religions to come together to exchange ideas, build relationships and learn about each others' cultures. Many believed that this increased cross-cultural understanding, in combination with communication, leadership, and critical thinking skills gained at JC:HEM, has helped students to reduce the number of interpersonal conflicts and the create more peaceful communities. In addition to increasing student's involvement within their communities, staff also believed JC:HEM is improving refugees' outlook on life and their future orientation, so that their futures seemed less bleak. These findings are particularly interesting, as research suggests that education can help facilitate good mental health indicators such as empowerment and positive outlook for refugees after displacement (Kirk, 2010; Zeus, 2011).

A significant challenge identified by staff centered around program delivery, and especially the difficulties encountered in communicating with faculty, either because of unclear channels, or faculty unresponsiveness or conflicting direction. Another main issue identified by staff is the entrenched gender bias from refugees' countries of origin, which in many cases prohibit women from full participation in education - a dynamic which implies the need to develop gender sensitive approaches to providing education (Anselme \& Hands, 2010).

\section{Limitations}

This study has limitations. Given the qualitative nature of the data, we are not able to generalize these findings to other populations of faculty and staff providing education to refugees. Data were only collected at one point in time and therefore may not capture the changing dynamics of online education delivery within and across sites. This study only reflects the perceptions of faculty and staff; students' perceptions are reported in other studies (Crea, 2016; Crea \& McFarland, 2015). We were not able to hear from all faculty and staff involved with JC:HEM, so it is possible that many responses provided in the context of this study are influenced by social desirability bias.

\section{Conclusion and implications}

As JC:HEM moves towards its next phase of implementation, with its new name of Jesuit Worldwide Learning (JWL, 2017), the program has expanded its scope to include other populations living at the margins, as well as refugees. Given the communication problems endemic to distance education - and which emerged in the JC:HEM pilot among faculty teams, between lead faculty and instructors, between instructors and on- 
site staff, and between instructors and students - increased efforts have been made to emphasize to lead faculty the importance of regular, clear communication with instructors and onsite staff. The Diploma curriculum and Community Service Learning Tracks have each been revised. Future efforts will focus on developing curricula through the lens of gender sensitivity (Anselme \& Hands, 2010), as a means of addressing the educational gender disparities that often arise from students' countries of origin.

Faculty also expressed some fatigue with the amount of time and effort required to teach a course on a voluntary basis, especially with the multiple challenges of teaching online in a cross-cultural context which are reflected in other studies of distance learning (Rogers et al., 2007). The findings of this study also suggest that, in terms of distance learning, the JC:HEM pilot faced similar challenges to other online programs, such as difficulty communicating with students (Boling et al., 2012; Ocak, 2011). A distinguishing feature of JC:HEM, however, is the use of onsite facilitators and on-line tutors that can help mediate online instruction, and help contextualize "static" online content. Through participation in online learning, with onsite mediated instruction, refugees and others at the margins have the potential to increase their digital capital (Seale et al., 2015) and mitigate social exclusion (de Haan, 1998). Importantly, through JC:HEM the voices of those at the margins are brought to the global classroom through online technology. Future studies should identify the specific outcomes to be achieved through online education for refugees - whether it is increased digital capital or greater social inclusion - and examine the extent to which these outcomes are fully realized.

The authors would like to thank the administrators, staff, and students of JC:HEM, as well as staff at Jesuit Refugee Service (JRS), for their assistance and participation in this study. The authors also thank students at the Boston College School of Social Work for assistance with analysis of qualitative data.
}

Funding

This study was funded by a research and evaluation contract from Jesuit Commons: Higher Education at the Margins (JC:HEM), awarded to the Boston College School of Social Work.

Authors' contributions

TC conceptualized and designed the study, collected the data, supervised data analysis and wrote the preliminary draft of the manuscript. NS assisted with data collection, served as the liaison with faculty respondents, and wrote portions of the final manuscript. Both authors read and approved the final manuscript.

Competing interests

Neither author has any competing interests in the publication of this study.

\section{Publisher's Note}

Springer Nature remains neutral with regard to jurisdictional claims in published maps and institutional affiliations.

\section{Author details}

${ }^{1}$ School of Social Work, Boston College, 140 Commonwealth Ave, Chestnut Hill, MA 02467, USA. ${ }^{2}$ Independent consultant, formerly with Jesuit Commons: Higher Education at the Margins (JC:HEM), Cambridge, United Kingdom.

Received: 5 September 2017 Accepted: 29 November 2017

Published online: 15 December 2017

References

Anselme, M. L., \& Hands, C. (2010). Access to secondary and tertiary education for all refugees: Steps and challenges to overcome. Refuge, 27(2), 3-7.

Bernard, R. M., de Rubalcava, B. R., \& St. Pierre, D. (2000). Collaborative online distance learning: Issues for future practice and research. Distance Education, 21(2), 260-277.

Bhatti, A., Tubaisahat, A., \& El-Qawasmeh, E. (2005). Using technology-mediated learning environment to overcome social and cultural limitations in higher education. Issues in Informing Science and Information Technology, 2, 67-76. 
Blessinger, P., \& Anchan, J. P. (2015). Democratizing higher education: International comparative perspectives. Routledge, New York.

Blin, F., \& Munro, M. (2008). Why hasn't technology disrupted academics' teaching practices? Understanding resistance to change through the lens of activity theory. Computers \& Education, 50, 475-490.

Boling, E. C., Hough, M., Krinsky, H., Saleem, H., \& Stevens, M. (2012). Cutting the distance in distance education: Perspectives on what promotes positive, online learning experiences. Internet and Higher Education, 15, 118-126.

Cook, R. G., Ley, K., Crawford, C., \& Warner, A. (2009). Motivators and inhibitors for university faculty in distance and e-learning. British Journal of Educational Technology, 40(1), 149-163.

Cooper, E. (2005). What do we know about out-of-school youths? How participatory action research can work for young refugees in camps. Compare: A Journal of Comparative Education, 35(4), 463-477.

Correa-Velez, I., Gifford, S. M., \& Barnett, A. G. (2010). Longing to belong: Social inclusion and wellbeing among youth with refugee backgrounds in the first three years in Melbourne, Australia. Social Science \& Medicine, 71, 1399-1408.

Crea, T. M. (2016). Refugee higher education: Contextual challenges and implications for program design, delivery, and accompaniment. International Journal of Educational Development, 46, 12-22.

Crea, T. M., \& McFarland, M. (2015). Higher education for refugees: Lessons from a 4-year pilot study. International Review of Education, 61(2), 235-245. https://doi.org/10.1007/s11159-015-9484-y.

Dankova, P., \& Giner, C. (2011). Technology in aid of learning for isolated refugees. Forced Migration Review, 38, 11-12.

de Haan, A. (1998). 'Social exclusion': An alternative concept for the study of deprivation? IDS Bulletin, 29(1), 10-19.

Easton, S. (2003). Clarifying the instructor's role in online distance learning. Communication Education, 52(2), 87-105.

Holley, D., \& Oliver, M. (2010). Student engagement and blended learning: Portraits of risk. Computers \& Education, 54, 693-700.

Jesuit Commons: Higher Education at the Margins (JC:HEM) (2010). Jesuit Commons: Higher Education at the Margins: vision and mission (white paper). Spokane: JC:HEM.

Jesuit Worldwide Learning (2017). Jesuit Worldwide Learning: Higher Education at the Margins. Available from: http://www.jwl. org/. Accessed 5 Sept 2017.

Kirk, J. (2010). Gender, forced migration and education: Identities and experiences of refugee women teachers. Gender and Education, 22(2), 161-176.

Krutka, D. G., Bergman, D. J., Flores, R., Mason, K., \& Jack, A. R. (2014). Microblogging about teaching: Nurturing participatory cultures through collaborative online reflection with pre-senvice teachers. Teaching and Teacher Education, 20, 83-93.

Liaw, M. (2006). E-learning and the development of intercultural competence. Language Learning and Technology, 10(3), 49-64.

McLoughlin, C., \& Oliver, R. (2000). Designing learning environments for cultural inclusivity: A case study of indigenous online learning at a tertiary level. Australian Journal of Educational Technology, 16(1), 58-72.

Ocak, M. A. (2011). Why are faculty members not teaching blended courses? Insights from faculty members. Computers \& Education, 56, 689-699.

Oncu, S., \& Cakir, H. (2011). Research in online learning environments: Priorities and methodologies. Computers \& Education, 57, 1098-1108.

Rhema, A., \& Miliszewska, I. (2010). Towards e-learning in higher education in Libya. Issues in Informing Science and Information Technology, 7, 423-437.

Rogers, P. C., Graham, C. R., \& Mayes, C. T. (2007). Cultural competence and instructional design: Exploration research into the delivery of online instruction cross-culturally. Educational Technology Research and Development, 55(2), 197-217.

Seale, J., Georgeson, J., Mamas, C., \& Swain, J. (2015). Not the right kind of 'digital capital'? An examination of the complex relationship between disabled students, their technologies, and higher education institutions. Computers \& Education, 82, 118-128.

Sparnon, N. (2011). JC:HEM interim outcome assessment report. Spokane: JC:HEM.

Strauss, A., \& Corbin, J. (1998). Basics of qualitative research: Techniques and procedures for developing grounded theory. Thousand Oaks, CA: Sage.

Volery, T., \& Lord, D. (2000). Critical success factors in online education. The International Journal of Educational Management, 14(5), 216-223.

Wright, L., \& Plasterer, W. (2010). Beyond basic education: Exploring opportunities for higher learning in Kenyan refugee camps. Refuge, 27(2), $42-57$.

Zeichner, K., Payne, K. A., \& Brayno, K. (2015). Democratizing teacher education. Journal of Teacher Education, 66(2), 122-135.

Zeus, B. (2011). Exploring barriers to higher education in protracted refugee situations: The case of Burmese refugees in Thailand. Journal of Refugee Studies, 24(2), 256-276.

\section{Submit your manuscript to a SpringerOpen ${ }^{\circ}$ journal and benefit from:}

- Convenient online submission

- Rigorous peer review

- Open access: articles freely available online

- High visibility within the field

- Retaining the copyright to your article

Submit your next manuscript at $\gg$ springeropen.com 